\title{
Comparative manufacture and cell-based delivery of antiretroviral nanoformulations
}

This article was published in the following Dove Press journal:

International Journal of Nanomedicine

16 December 201I

Number of times this article has been viewed

\author{
Shantanu Balkundi' \\ Ari S Nowacek' \\ Ram S Veerubhotla' \\ Han Chen ${ }^{2}$ \\ Andrea Martinez-Skinner ${ }^{1}$ \\ Upal Roy' \\ R Lee Mosley ${ }^{1,3}$ \\ Georgette Kanmogne' \\ Xinming Liu ${ }^{1,3,4}$ \\ Alexander $\mathrm{V}$ Kabanov ${ }^{3,4}$ \\ Tatiana Bronich ${ }^{3,4}$ \\ JoEllyn McMillan' \\ Howard E Gendelman 1,3 \\ 'Department of Pharmacology \\ and Experimental Neuroscience, \\ University of Nebraska Medical \\ Center, Omaha, NE, USA; ${ }^{2}$ Center \\ for Biotechnology, University \\ of Nebraska-Lincoln, Lincoln, NE, \\ USA $;{ }^{3}$ Center for Drug Delivery \\ and Nanomedicine, University \\ of Nebraska Medical Center, \\ Omaha, NE, USA; ${ }^{4}$ Department \\ of Pharmaceutical Sciences, College \\ of Pharmacy, University of Nebraska \\ Medical Center, Omaha, NE, USA
}

Correspondence: Howard E Gendelman Department of Pharmacology and Experimental Neuroscience, 985880 Nebraska Medical Center Omaha, NE 68198-5880, USA

$\mathrm{Tel}+\mathrm{I} 4025598920$

Fax + I 4025593744

Email hegendel@unmc.edu
Abstract: Nanoformulations of crystalline indinavir, ritonavir, atazanavir, and efavirenz were manufactured by wet milling, homogenization or sonication with a variety of excipients. The chemical, biological, immune, virological, and toxicological properties of these formulations were compared using an established monocyte-derived macrophage scoring indicator system. Measurements of drug uptake, retention, release, and antiretroviral activity demonstrated differences amongst preparation methods. Interestingly, for drug cell targeting and antiretroviral responses the most significant difference among the particles was the drug itself. We posit that the choice of drug and formulation composition may ultimately affect clinical utility.

Keywords: human immunodeficiency virus type one, nanotoxicology, monocyte-derived macrophage, nanoformulated antiretroviral therapy, manufacturing techniques

\section{Introduction}

Antiretroviral therapy (ART) has profoundly reduced disease morbidity and mortality among human immunodeficiency virus (HIV) infected individuals worldwide. ${ }^{1-5}$ However, the short half-life of the drugs necessitates multiple daily dosing schedules limiting both access and compliance. Moreover, failure of ART penetration into sanctuaries of persistent viral replication including the lymphoreticular and central nervous systems, and a range of secondary drug-induced toxicities further highlight the limitations of chronic drug treatment regimens. ${ }^{6-10}$ One approach to address such limitations is through long-acting antiretroviral drug nanoformulations with half-lives measured in week(s) rather than hours. To this end, improvements in drug pharmacodynamics were described for ART nanoformulations (nanoART). ${ }^{4,11,12}$ Prior works demonstrated that such nanoART could be carried in monocyte-macrophages and reach viral sanctuaries. ${ }^{11-15}$ These studies also showed that size, shape, and charge of crystalline indinavir (IDV), ritonavir (RTV), atazanavir (ATV), and efavirenz (EFV) affect uptake, release, cytotoxicities, and antiretroviral responses. ${ }^{11,15-17}$ However, absent from prior investigations was an analysis of the method of particle manufacture.

Crystalline nanoformulations ${ }^{11,16,18-24}$ prepared by sonication, wet milling and high-pressure homogenization differ in how surfactant-encapsulated drug crystals are produced. Wet milling produces particles by fractionating the surfactant-coated drug crystals to smaller sizes through impaction with micron-sized ceramic/zirconium beads. ${ }^{24-26}$ Homogenization elicits particle suspensions by passing surfactant-coated crystals through a narrow bore under high pressure (1500-20,000 psi). ${ }^{13,18,23}$ Sonication results in spherical poly(D,L-lactide-co-glycolide) (PLGA) nanoparticles (NP), 
in which the drug is dissolved in a polymer solution, then agitated by ultrasonication to obtain individual nanosized droplets. ${ }^{21,27,28}$ Such techniques can also produce unexpected findings in regard to particle integrity and its effects on the drug-carrier target cell. In addition, micelles can also be used as nanocarriers. However, these particles are smaller in size than the crystalline formulations and drug loading is much more limited. Different methods of nanoART construction can dramatically alter the particle's physical properties and how they interact with carrier cells; thus, production methods can affect the ultimate translation of the final clinical formulations.

\section{Materials and methods}

\section{Preparation and characterization of nanoART}

RTV and EFV were obtained in the free base form from Shengda Pharmaceutical Co (Zhejiang, China) and Hetero Labs, Ltd (Hyderabad, India). The sulfate forms of IDV and ATV were purchased from Longshem Co (Shanghai, China) and Gyma Laboratories of America Inc (Westbury, NY), respectively. The free bases of IDV and ATV were made using a $1 \mathrm{~N} \mathrm{NaOH}$ solution. The surfactants used in this study were: poloxamer-188 (P188; Sigma-Aldrich, St Louis, MO), 1,2-distearoyl-phosphatidylethanolamine-methylpolyethyleneglycol conjugate-2000 ( $\mathrm{mPEG}_{2000} \mathrm{DSPE}$ (Genzyme Pharmaceuticals LLC, Cambridge, MA), and sodium dodecyl sulfate (SDS) (Bio-Rad Laboratories, Hercules, CA). These were suspended in $10 \mathrm{mM}$ HEPES, $\mathrm{pH} 7.8$, and free base drug was added ( $0.6 \%$ by weight). Combinations of drug and surfactant were (1) ATV in 0.5\% P188; (2) IDV in $0.5 \% \mathrm{P} 188$ and $0.5 \% \mathrm{SDS}$; (3) RTV or EFV in $0.3 \% \mathrm{P} 188$ and $0.1 \% \mathrm{mPEG}_{2000} \mathrm{DSPE}$. A homogeneous dispersion was formed by agitation with an Ultraturrax T-18 rotor-stator mixer (IKA ${ }^{\circledR}$ Works Inc, Wilmington, NC). For preparation of nanosuspensions by wet milling, mixtures were transferred to a NETZSCH MicroSeries Wet Mill (NETZSCH Premier Technologies, LLC, Exton, PA) with $50 \mathrm{~mL}$ of $0.8 \mathrm{~mm}$ grinding media (zirconium ceramic beads, Saint-Gobain ZirPro, La Pontet, France). The sample was milled for 30 minutes to 1 hour at speeds ranging from 600 to $4320 \mathrm{rpm}$ until desired particle size was achieved. ${ }^{11,16}$ For preparation of suspensions by homogenization, mixtures were transferred to an Avestin C5 high-pressure homogenizer (Avestin Inc, Ottawa, ON) and extruded at 20,000 pounds per square inch for $\sim 30$ passes or until the desired particle size was attained. ${ }^{15,16}$ Particle size, polydispersity (PDI), and surface charge (zeta potential) were determined by dynamic light scattering (DLS) using a Malvern Zetasizer Nano Series Nano-ZS (Malvern Instruments Inc, Westborough, MA). A Hitachi S4700 FieldEmission Scanning Electron Microscope (Hitachi High Technologies America Inc, Schaumburg, IL) was used to evaluate particle morphology and validate DLS tested particle size. After the desired particle size was achieved, samples were centrifuged at $10,000 \times g$ for 30 minutes at $4^{\circ} \mathrm{C}$. The resulting pellet was resuspended in surfactant solution containing 9.25\% sucrose to adjust tonicity. Drug concentration in the final suspension was determined using reversephase high-performance liquid chromatography (RP-HPLC) as previously described. ${ }^{15}$

For manufacturing NP using sonication, $6 \mathrm{~g}$ of PLGA, (RESOMER RG752H; Sigma-Aldrich) was added to $50 \mathrm{~mL}$ dichloromethane (HPLC-grade) and mixed until complete dissolution. Drug crystals (1.25 g) were added to the dichloromethane/PLGA solution and mixed to obtain complete dissolution. This solution was added to a $1 \%$ polyvinyl alcohol (PVA; Sigma-Aldrich) surfactant solution cooled in an ice bath, and then sonicated using a Cole Parmer Ultrasonic processor (Vernon Hills, IL) at 50\% amplitude for $10 \mathrm{~min}$ utes. Particle size was determined by dynamic light scattering using a Zetasizer. The sonication time was increased at 2-minute intervals up to a maximum of 16 minutes total if the particle size was greater than $1.5 \mu \mathrm{m}$. The samples were characterized by light microscopy (20× magnification). The remaining suspension was vortexed and mixed at an adequate speed overnight at room temperature, then collected after 24 hours and centrifuged step-wise at $8100 \times g$ for 20 minutes at $5^{\circ} \mathrm{C}$. After decanting the supernatant, the pellet was resuspended in $75 \mathrm{~mL}$ of filtered, reverse osmosis (RO) water and the samples centrifuged again at $8100 \times g$ for 20 minutes at $5^{\circ} \mathrm{C}$. The pellet was resuspended in $1 \%$ mannitol (Sigma-Aldrich) in RO water for lyophilization. The particle size was again measured using a Zetasizer and drug concentration determined by RP-HPLC. ${ }^{16}$

\section{Human monocyte isolation and cultivation}

Human monocytes were obtained by leukapheresis from HIV-1 and hepatitis B seronegative donors and purified by counter-current centrifugal elutriation. Cell purity was greater than $96 \%$ as determined by immunolabeling with anti-CD68 (clone KP-1) from Wright-stained cytospins. Monocytes were cultured in Dulbecco's Modified Eagles Medium (DMEM) supplemented with 10\% heat-inactivated human serum, $1 \%$ glutamine, $50 \mu \mathrm{g} / \mathrm{mL}$ gentamicin, $10 \mu \mathrm{g} / \mathrm{mL}$ ciprofloxacin, and $1000 \mathrm{U} / \mathrm{mL}$ recombinant 
human macrophage-colony stimulating factor (MCSF) (a generous gift from Pfizer Inc, Cambridge, MA) at a cell density of $1 \times 10^{6}$ cells $/ \mathrm{mL}$ at $37^{\circ} \mathrm{C}$ in a $5 \% \mathrm{CO}_{2}$ humidified atmosphere. Monocytes differentiated into monocyte-derived macrophages (MDM) after 7 days of culture. ${ }^{29}$

\section{Electron microscopy}

For scanning electron microscopy (SEM) of the nanoparticles, $10 \mu \mathrm{L}$ of nanosuspension was diluted in $1.5 \mathrm{~mL}$ of $0.2 \mu \mathrm{m}$-filtered double distilled water. The diluted suspension was mixed, and a $50 \mu \mathrm{L}$ aliquot was transferred to a filtration apparatus (Swinnex 13 polypropylene filter holder, Millipore, Billerica, MA) assembled with a $0.2 \mu \mathrm{m}$ pre-wetted polycarbonate filter membrane (Nuclepore Track-Etched, Whatman International Ltd, Kent, ME). The entire solution volume was pulled through the filtration membrane by vacuum. The membrane was washed with $500 \mu \mathrm{L}$ of filtered double-distilled water. The membrane was allowed to dry for 24 hours, fixed to an aluminum pin stub using double stick conductive carbon tape, and sputter coated with palladium (EMITECH K575X; Quorum Technologies, Ashford, Kent, UK). The lyophilized PLGA NP were fixed to the double stick conductive carbon tape surface and sputter coated with palladium before imaging. The samples were affixed to the specimen stub and imaged using a Hitachi S4700 Field-Emission Scanning Electron Microscope (Hitachi High Technologies America Inc, Schaumburg, IL).

\section{NanoART uptake and release kinetics}

MDM uptake, retention, and release of nanoART were determined as previously described. ${ }^{15}$ MDM were incubated with $100 \mu \mathrm{M}$ nanoART and cell uptake determined over an 8-hour period. Adherent MDM were washed three times with phosphate buffered saline (PBS) and scraped into $1 \mathrm{~mL}$ PBS. Cells were pelleted by centrifugation at $950 \times g$ for 10 minutes at $4{ }^{\circ} \mathrm{C}$ and the supernatant was removed. Cell pellets were resuspended in $200 \mu \mathrm{L}$ of HPLC-grade methanol, sonicated, and centrifuged at $20,000 \times g$ for 10 minutes at $4^{\circ} \mathrm{C}$. The methanol extract was stored at $-80^{\circ} \mathrm{C}$ until drug analysis. For determination of cell retention and release of nanoART, MDM were exposed to $100 \mu \mathrm{M}$ nanoART for 8 hours, washed three times with PBS, and fresh medium added. MDM were cultured for up to 15 days with half medium exchanges every other day for all treatment assays. On days 1, 5, 10, and 15 following nanoART treatment, MDM were collected and methanol extracts prepared. Replicate media samples were collected at each time point. Both cell extract and media samples were stored at $-80^{\circ} \mathrm{C}$ until processed and analyzed for drug by HPLC as previously described. ${ }^{15}$ Duplicate $20 \mu \mathrm{L}$ injections of processed cells or media were analyzed by RP-HPLC using a YMC-Pack Octyl C8 column (Waters Inc, Milford, MA) with a C8 guard cartridge. Analytes were eluted using a mobile phase of $48 \%$ acetonitrile $/ 52 \% 25 \mathrm{mM}$ $\mathrm{KH}_{2} \mathrm{PO}_{4}, \mathrm{pH} 4.15$, at a flow rate of $0.4 \mathrm{~mL} /$ minute and were detected by UV detection at $212 \mathrm{~nm}$. Drug levels were quantitated by comparison of peak area to a standard curve of free $\operatorname{drug}(0.025-100 \mu \mathrm{g} / \mathrm{mL})$ in methanol.

\section{HIV-I infection}

MDM were treated with $100 \mu \mathrm{M}$ nanoART for 8 hours, washed to remove excess drug, then given fresh medium without drug. At days 1, 5, 10, and 15 following nanoART treatment, the cells were infected with HIV-1 ${ }_{\mathrm{ADA}}$ at a multiplicity of infection (MOI) of 0.01 infectious viral particles/cell. ${ }^{29}$ Following 24-hours of infection, virus was washed away and medium was replaced with fresh virusfree medium. Cells were cultured for an additional 10 days with half medium exchanges every other day. At this time, medium samples were collected for measurement of reverse transcriptase (RT) activity and cells were fixed in 4\% paraformaldehyde (PFA) for determination of HIV-1p24 antigen expression.

\section{Measurement of RT activity}

RT activity in cell medium samples was determined as previously described. ${ }^{12}$ Cell supernatant samples $(10 \mu \mathrm{L})$ were mixed with $10 \mu \mathrm{L}$ of $100 \mathrm{mM}$ Tris- $\mathrm{HCl}$ (pH 7.9), $300 \mathrm{mM}$ $\mathrm{KCl}, 10 \mathrm{mM}$ DTT, and $0.1 \%$ nonyl phenoxylpolyethoxylethanol-40 (NP-40) in a 96-well plate. The samples were incubated at $37^{\circ} \mathrm{C}$ for 15 minutes. Twenty-five microliters of a solution containing $50 \mathrm{mM}$ Tris- $\mathrm{HCl}$ (pH 7.9), $150 \mathrm{mM} \mathrm{KCl}$, $5 \mathrm{mM}$ DTT, $15 \mathrm{mM} \mathrm{MgCl}, 0.05 \% \mathrm{NP}-40,10 \mu \mathrm{g} / \mathrm{mL}$ poly(A), $0.250 \mathrm{U} / \mathrm{mL}$ oligo $\mathrm{d}(\mathrm{T}),{ }^{12-18}$ and $10 \mu \mathrm{Ci} / \mathrm{mL}{ }^{3} \mathrm{H}-\mathrm{TTP}$ was then added to each well and incubated at $37^{\circ} \mathrm{C}$ for 18 hours. Following incubation, $50 \mu \mathrm{L}$ of ice-cold $10 \%$ trichloroacetic acid was added to each well, and the well contents were harvested onto glass fiber filters and assessed for ${ }^{3} \mathrm{H}$-TTP incorporation by $\beta$-scintillation spectroscopy. ${ }^{30}$

\section{HIV-I 24 antigen immunostaining}

HIV-1p24 antigen staining was determined in PFA-fixed cells as previously described. ${ }^{12}$ Mouse monoclonal antibodies to HIV-1 p24 (1:100, Dako, Carpinteria, CA) were used to detect HIV-1 infected cells. Binding of p24 antibody was detected using Dako EnVision+ System-HRP labeled polymer antimouse secondary antibody and diaminobenzidine staining. 
Images were acquired using a Nikon TE300 microscope with a $20 \times$ objective (Nikon, Tokyo, Japan).

\section{Cytotoxicity}

For determination of cytotoxicity, MDM were cultured at a concentration of $6.25 \times 10^{5}$ cells $/ \mathrm{mL}$ and treated with nanoformulations of IDV, RTV, or EFV at $0.1 \mathrm{mM}$ for 12 hours at $37^{\circ} \mathrm{C}$ in $5 \% \mathrm{CO}_{2}$. Following loading of each nanoformulation, cells were washed with serum-free culture medium to remove excess NP and cytotoxicity assessed over the subsequent 24 hours using the alamarBlue ${ }^{\mathrm{TM}}$ assay (AbD Serotec, Raleigh, NC) according to the manufacturer's instructions.

\section{Statistical analyses}

Data analyses were carried out using Prism (GraphPad Software Inc, La Jolla, CA). Significant differences in cytotoxicity response were determined by one-way ANOVA followed by Bonferroni's multiple comparisons test. $P$ values for significance are indicated.

\section{Results}

\section{Characterization}

Nanoformulations of antiretroviral drugs were prepared by wet milling, homogenization, and sonication. Dynamic light scattering was used to determine particle size, polydispersity index (PDI), and surface charge (zeta potential). Wet milled and homogenized formulations for each drug contained identical surfactants and were similar in size and charge (Table 1). The IDV nanoformulations, M1004 and H1004, were the most diverse with sizes of 252 and $418 \mathrm{~nm}$ and charges of -40.6 and $-15.1 \mathrm{mV}$, respectively, for those milled and homogenized particles. P1001 was $366.6 \mathrm{~nm}$ in size, smaller than the nanocrystals, but had a PDI of 0.451 . The charge of P1001 ( $-9.57 \mathrm{mV})$ was lower than M1004 and H1004. The PDI of the nanocrystal formulations ranged from 0.152 for M2006 to 0.295 for H4002, indicating a size diversity of the NP. The NP formed by sonication of ATV, RTV, and EFV were consistent in size and PDI.

Scanning electron microscopy (SEM) at 15,000× magnification was used to visualize nanoART morphologies, This approach showed that the IDV crystals appeared as short rods with rough edges (Figure 1). The ATV crystals formed longer thin rods with smooth edges. RTV nanocrystals formed short thick rods. EFV particles were ellipsoid. Overall, the NP manufactured with milling had rougher edges than those made by homogenization. All NP manufactured with sonication using PLGA were spherical.

\section{NanoART uptake by MDM}

MDM uptake of wet milled and homogenized nanoformulations was compared for each drug during an 8-hour time interval (Figure 2). Previous studies have shown that levels of drug in MDM exposed to crystalline nanoformulations of IDV, RTV, ATV, or EFV are more than $90 \%$ of maximum levels by 8 hours. ${ }^{12,15}$ At 2, 4, and 8 hours, MDM uptake of homogenized nanoformulations of IDV and EFV was greater than observed with wet milled formulations. At 8 hours, IDV and EFV levels in MDM were 1.4-fold higher with homogenized compared to wet milled formulations. In contrast, uptake of H2006 and H3001 was less than for M2006 and M3001, respectively. At 8 hours, drug levels of RTV in MDM were 11.3 vs $9.3 \mu \mathrm{g} / 10^{6}$ cells for M2006 and H2006, respectively. Similarly, levels of ATV were 36.6 vs $29.6 \mu \mathrm{g} / 10^{6}$ cells for

Table I Physicochemical characteristics of nanoformulations of antiretroviral drugs

\begin{tabular}{|c|c|c|c|c|c|}
\hline Drug & Formulation designation & Surfactant & Size $(\mathrm{nm})^{\mathrm{a}}$ & PDI $^{\mathrm{b}}$ & Zeta potential $(\mathrm{mV})$ \\
\hline \multirow[t]{3}{*}{ Indinavir } & IDV-MI004 & PI88, SDS & 252 & 0.286 & -40.58 \\
\hline & IDV-HI004 & PI88, SDS & 418 & 0.239 & -15.09 \\
\hline & IDV-PI00I & PLGA, PVA & 367 & 0.451 & -9.57 \\
\hline \multirow[t]{3}{*}{ Ritonavir } & RTV-M2006 & $\mathrm{PI} 88, \mathrm{mPEG}_{2000} \mathrm{DSPE}$ & 374 & 0.152 & -30.11 \\
\hline & RTV-H2006 & $\mathrm{PI} 88, \mathrm{mPEG}_{2000} \mathrm{DSPE}$ & 405 & 0.220 & -28.79 \\
\hline & RTV-P200I & PLGA, PVA & 302 & 0.22 & -26.10 \\
\hline \multirow[t]{3}{*}{ Atazanavir } & ATV-M300I & PI88 & 281 & 0.288 & $-|5.3|$ \\
\hline & ATV-H300I & PI88 & 314 & 0.200 & -31.65 \\
\hline & ATV-P300I & PLGA, PVA & 270 & 0.148 & -26.40 \\
\hline \multirow[t]{3}{*}{ Efavirenz } & EFV-M4002 & PI88, mPEG ${ }_{2000}$ DSPE & 325 & 0.281 & -32.47 \\
\hline & EFV-H4002 & $\mathrm{PI} 88, \mathrm{mPEG}_{2000} \mathrm{DSPE}$ & 388 & 0.295 & -24.43 \\
\hline & EFV-P400I & PLGA, PVA & 288 & 0.198 & -31.00 \\
\hline
\end{tabular}

Notes: ${ }^{a}$ The particle sizes; ${ }^{b}$ polydispersity indices (PDI) were determined by dynamic light scattering; the z-average diameters are presented.

Abbreviations: ATV, atazanavir; DSPE, I,2-distearoyl-phosphatidyl-ethanolamine; EFV, efavirenz; IDV, indinavir; mPEG, methyl-polyethylene-glycol; PI88, poloxamer I88; PLGA, poly(lactic-co-glycolic acid); PVA, polyvinylalcohol; RTV, ritonavir; SDS, sodium dodecyl sulfate. M, wet milled; P, PLGA; H, homogenized. 

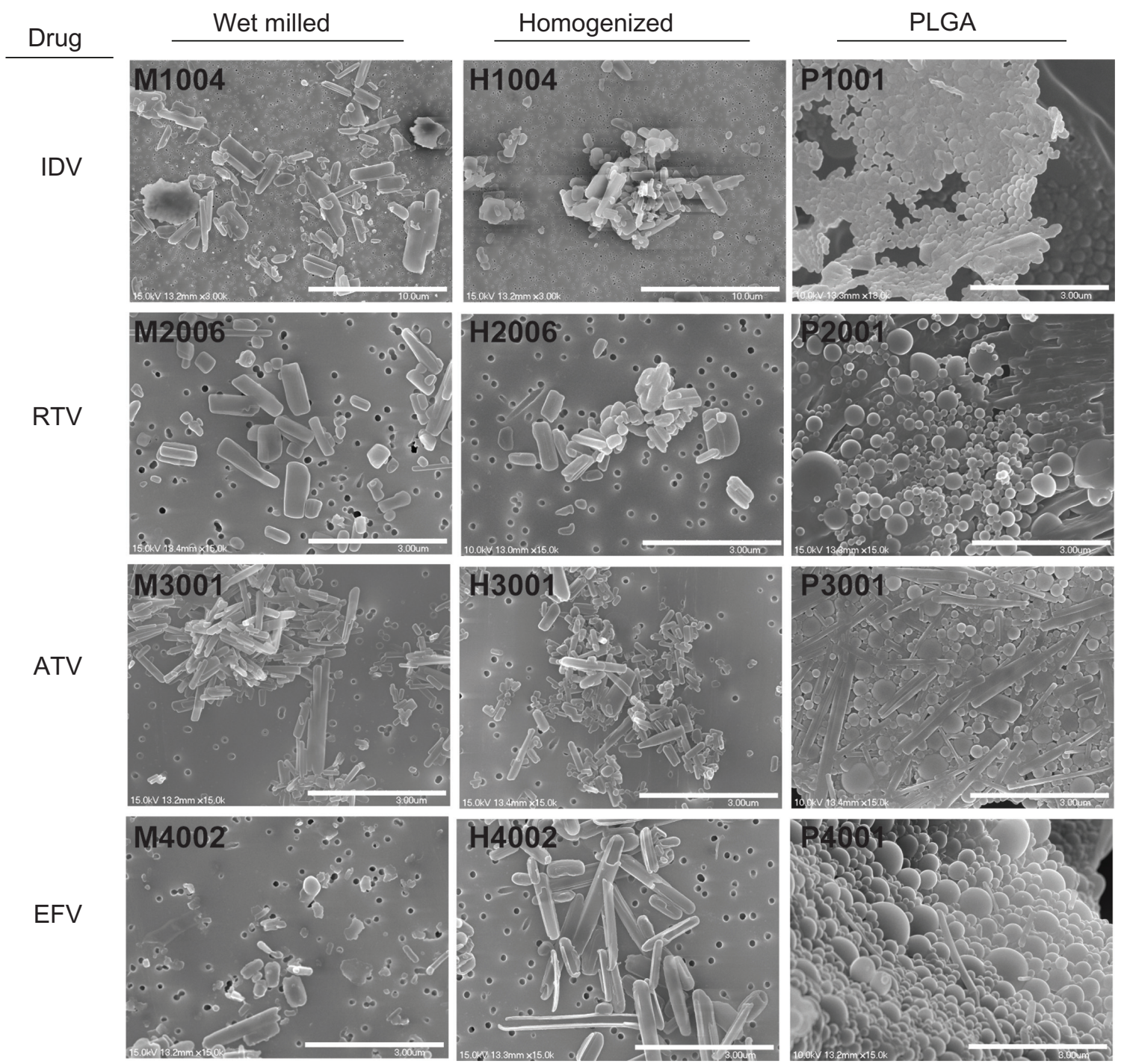

Figure I Scanning electron microscopy (SEM) images of nanoART morphology.

Notes: SEM analysis (magnification, 15,000x) of nanoformulated IDV, RTV, ATV, and EFV produced by homogenization, wet-milling, and sonication on top of a $0.2 \mu \mathrm{m}$ polycarbonate filtration membrane. Scale bar equals $10 \mu \mathrm{m}$ for IDV and $3 \mu \mathrm{m}$ for RTV, ATV and EFV. Wet milling and homogenization show crystalline nanoparticles while sonicated formulations are seen as spherical nanoparticles.

Abbreviations: IDV, indinavir; RTV, ritonavir; ATV, atazanavir; EFV, efavirenz.

M3001 and H3001, respectively. The uptake of P1001 was higher than M1004 and H1004 at 2 hours and 1.2 times lower than homogenized NP at 4 hours. Uptake of P3001 was lower than for both M3001 and H3001. ATV concentration in cells after 8 hours $\left(8.1 \mu \mathrm{g} / 10^{6}\right.$ cells $)$ was 3 - to 4 -fold lower following P3001 treatment than that for homogenized or milled particles ( 29.6 and $36.6 \mu \mathrm{g} / 10^{6}$ cells, respectively). P2001 was rapidly taken up over the first hour and at 8 hours was $6.6 \mu \mathrm{g} / 10^{6}$ cells compared to $9.4 \mu \mathrm{g} / 10^{6}$ cells and $11.3 \mu \mathrm{g} / 10^{6}$ cells for H2006 and M2006, respectively. For EFV, the uptake of P4001 was $~ 2$-fold lower than for M4001 and $\mathrm{H} 4001$ and at 8 hours was $0.6 \mu \mathrm{g} / 10^{6}$ cells vs 1.0 and $1.5 \mu \mathrm{g} / 10^{6}$ cells, for M4001 and H4001, respectively.

\section{NanoART retention and release by MDM}

Retention profiles within cells were similar for wet milled, homogenized, and PLGA formulations of the four drugs (Figure 2). The cellular drug content for all IDV formulations decreased by $>85 \%$ over 24 hours and was undetectable by day 5 . This was also observed for EFV formulations although limited drug levels were present at day 10 . The concentration of the drug was lower at days 1 and 5 for P2001 compared to M2006 and H2006. Drug levels retained with all ATV formulations was significantly higher than the others manufactured using all three techniques. The amount of drug retained after 15 days was $\sim 10 \mu \mathrm{g} / 10^{6}$ cells for M3001 and $\mathrm{H} 3001$ and $\sim 2 \mu \mathrm{g} / 10^{6}$ cells for $\mathrm{P} 3001$. 
Uptake
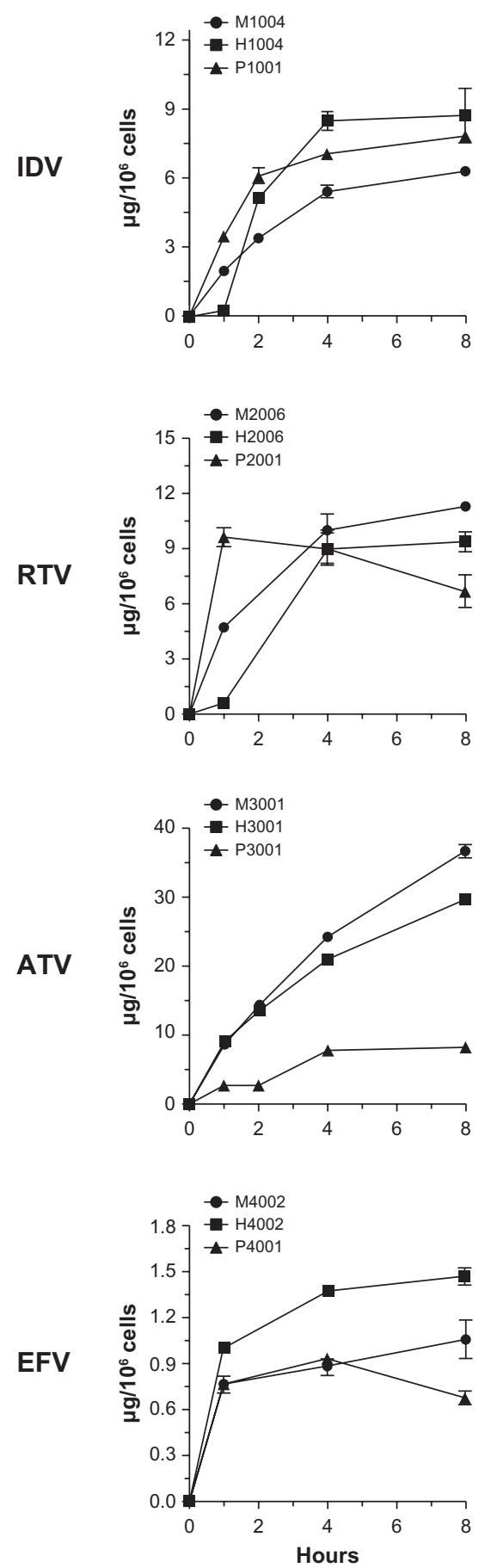

Retention
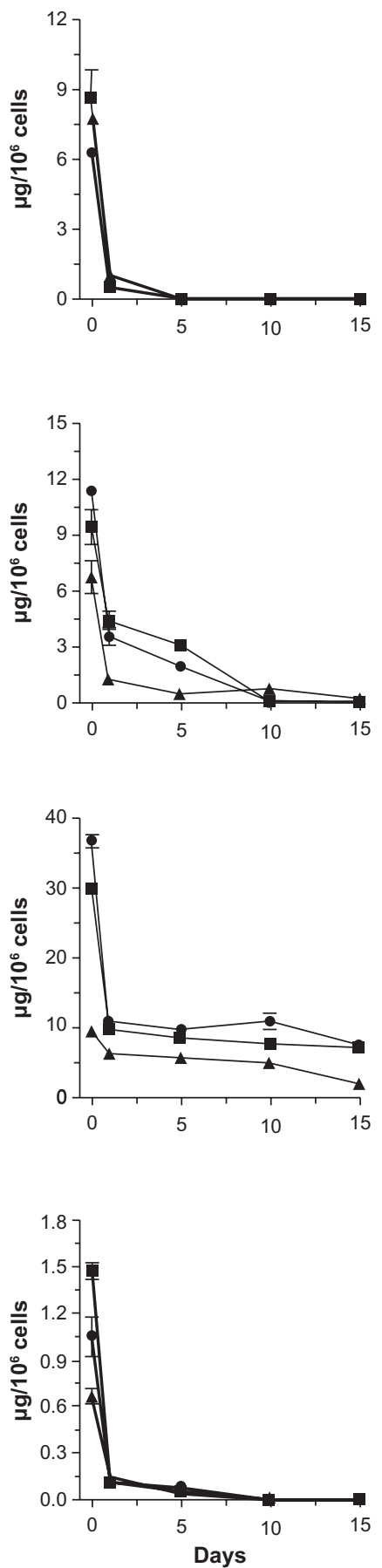

Release
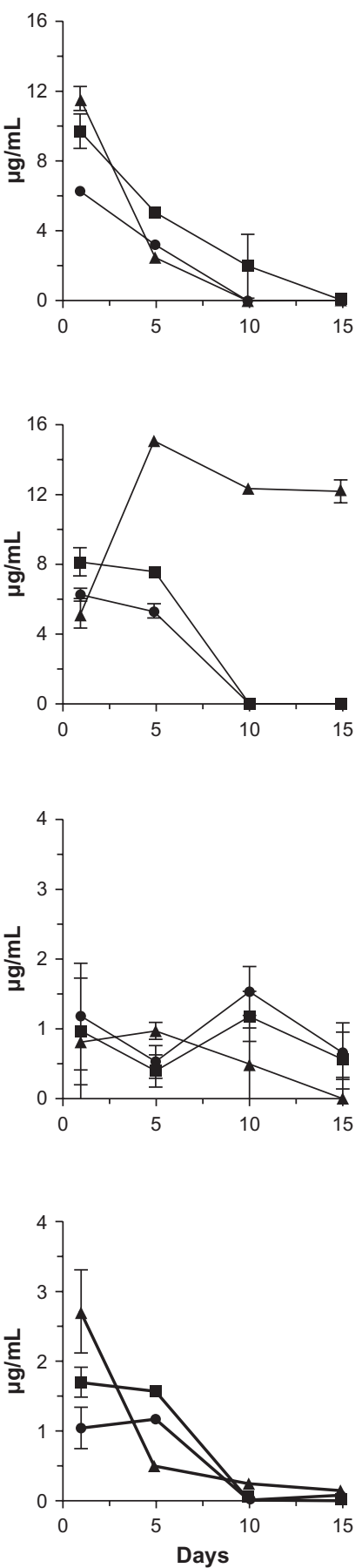

Figure 2 Macrophage uptake, retention and release of nanoART.

Notes: Time courses of monocyte-derived macrophage (MDM) uptake of IDV, RTV, ATV, and EFV nanoparticles manufactured by wet-milling, homogenization or sonication over a period of 8 hours and cell retention and release over a period of 15 days after treatment. Data represent the mean \pm SEM for $\mathrm{n}=3$ determinations per time.

Retention levels of IDV and EFV in MDM fell to less than $10 \%$ of loading levels by 24 hours after medium exchanges, were minimal at 5 days, but were undetectable by 10 days. Levels of RTV fell 65\%-70\% during the first 24 hours after cell loading, were $15 \%-30 \%$ of loading levels at 5 days, and were undetectable by 10 days. The cell levels of P2001 formulations were also reduced by $70 \%$ in the first 24 hours and were $10 \%$ of loading levels at 5 and 10 days, but were undetectable at day 15. Cellular levels of ATV dropped 70\% during the first 24 hours then remained at $20 \%$ of loading 
levels through 15 days. This observation is significant as ATV is a leading drug in the clinic. ${ }^{31,32}$

While initial medium levels ( 24 hour post-treatment) were variable between wet milled or homogenized and PLGA formulations, the rate of decline was similar for wet milled and homogenized formulations. Medium levels at day 1 were higher for H1004 than for M1004, but the rates of decline were similar for both formulations. RTV levels in the medium were also higher for H2006 than for M2006 at 1 day, but levels of both formulations only slightly declined over the next 5 days. By 10 days, medium levels were low to undetectable for either RTV formulation. Levels of ATV release were similar for both M3001 and H3001. Medium levels of ATV remained steady from 1 to 15 days. Medium levels of EFV were also higher for H4002 than for M4002 at 1 day. Similar to the profile seen for RTV, the medium levels of EFV only slightly declined over the next 5 days, but then fell to undetectable levels by 10 days. For the PLGA formulations, there were sharp declines of drug release. The drug levels reduced by a total of more than $80 \%$ in 5 days and were undetectable at day 10 with the exception of P2001 at day 5. The medium level remained almost the same for P3001 over the first 5 days, was reduced by $50 \%$ at 10 days and was undetectable on day 15 . P4001 levels in media reduced by $80 \%$ by 5 days, an additional $10 \%$ by 10 days, and were undetectable by day 15 .

\section{NanoART-induced antiretroviral activities}

Antiretroviral efficacy was assessed by measuring RT activity and HIV-1p24 following one exposure of MDM to nanoART and serial-timed infections (1-15 days) with HIV-1 ${ }_{\mathrm{ADA}}$. Viral profiles were similar for milled, homogenized and PLGA formulations of each drug when determined by RT activity (Figure 3). All formulations, both crystalline and PLGA, suppressed viral replication by $>87 \%$ when viral challenge was made 1 day after nanoART treatment. This suppression was reduced for viral challenges at 5 to 15 days after nanoART treatment for IDV, RTV, and ATV. For IDV formulations, suppression was reduced from $44 \%$ for $\mathrm{H} 1004$ on viral challenge day $5 \%$ to $23 \%$ and $21 \%$ on days 10 and 15 , respectively. Suppression over time was similar for M1004, at 33\% on day 5 , declining to $28 \%$ on day 10 , and reaching $21 \%$ on day 15 . The viral suppression for P1001 was greater than for M1004 or H1004 and ranged from $74 \%$ at day 5 to $54 \%$ by day 15. For RTV formulations, the profiles were very similar for M2006 and H2006, with viral suppression greater than $83 \%$ on day 5 . Suppression was reduced to $<32 \%$ on day 10 and by day 15 was only $24 \%$. In contrast, P2006 suppression remained $>67 \%$ for days 5,10 , and 15 . For all ATV formu- lations, viral suppression was $>70 \%$ when challenged on days 5,10 , or 15 after nanoART was administered to the MDM. EFV formulations were the most effective at suppressing viral replication. All EFV formulations suppressed viral expression by $>87 \%$ on all challenge days including day 15 . These results were mirrored by immunostaining of HIV-1p24 antigen (Figure 4). In general, a time-dependent increase in HIV-1p24 antigen was observed from challenge days 1 through 15. At all timepoints, immunostains of EFV nanoformulation-treated MDM administered prior to viral infection were similar to uninfected cells.

\section{Cytotoxicity tests}

To determine whether uptake and retention of nanoformulations would be detrimental to MDM, we evaluated cytotoxicity of eight independent NP manufactured by two different methods, homogenization and wet milling, using the alamarBlue $^{\mathrm{TM}}$ redox assay. These included four homogenized (two RTV, one ATV, and one EFV) and four wet milled (two RTV, one ATV, and one EFV) formulations. alamarBlue ${ }^{\mathrm{TM}}$ redox assay tests were performed on human MDM using each formulation administered at a dose of $0.1 \mathrm{mM} .^{17}$ Cytotoxicity was determined at 24 hours and demonstrated that homogenized RTV nanoformulations induced modest changes ( $15 \%$ decrease at 24 hours) in MDM viability compared to control cells. For milled RTV nanoformulations, while M2006 induced no significant change in alamarBlue ${ }^{\mathrm{TM}}$ reduction compared to control cells at 24 hours, M2001 significantly decreased MDM viability by $53 \%$ at 24 hours (Figure 5A). ATV nanoformulations did not significantly affect cell viability compared to control cells (Figure 5B), while EFV nanoformulations decreased cell viability by 30\%-67\% (Figure 5C). Fluconazole nanoparticles manufactured in parallel as exipient controls showed no effect on MDM viability and function. ${ }^{17}$

\section{Discussion}

We prepared NP using poorly water-soluble antiretroviral drugs by wet milling, homogenization, and sonication, and studied differences in nanoART responses. We used a cellbased screening approach to perform a direct comparison of nanoformulations of antiretroviral drugs produced by a variety of manufacturing techniques. We did this in order to assess which formulations could be further developed as long-acting injectables for animal and inevitably clinical studies. IDV-, RTV-, ATV-, and EFV-NP coated with the same excipients were compared for size, shape, and charge. The IDV wet milled and homogenized NP were diverse in 

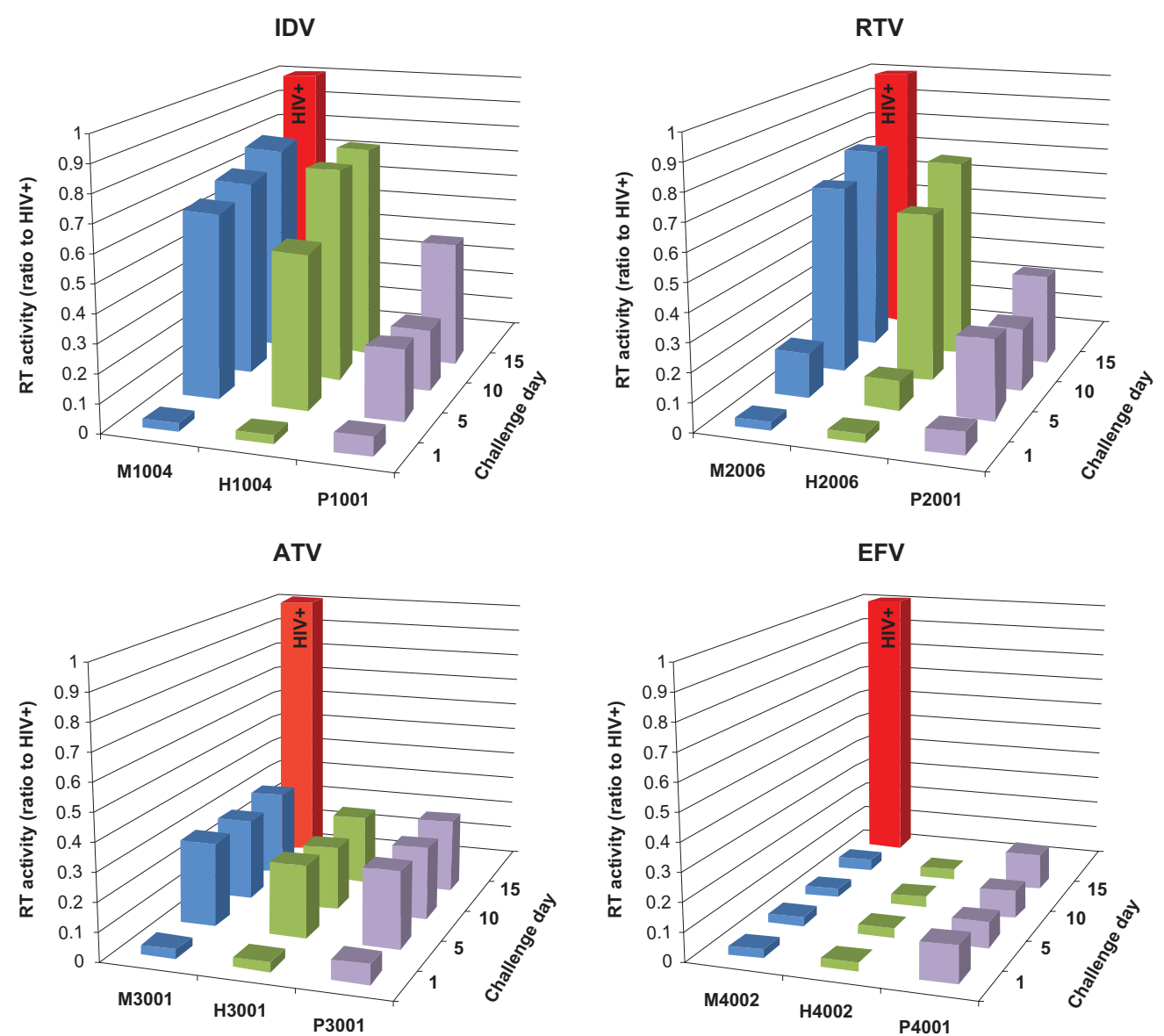

Figure 3 Comparison of antiretroviral effects of various formulations of IDV, RTV, ATV, and EFV as measured by reverse transcriptase (RT) activity. Notes: Monocyte-derived macrophages (MDM) were treated with $100 \mu \mathrm{M}$ of nanoparticles for 8 hours. MDM were infected with HIV-I ${ }_{\text {ADA }}$ on days I, 5 , I0, and I5 post-loading and cultured for 10 days after infection. Medium was removed and RT activity was measured by ${ }^{3} \mathrm{H}$-TTP incorporation. Data are normalized to activity in HIV-I infected untreated cells cultured in parallel. Data represent mean \pm SEM for $n=4$ determinations per treatment.

size. Indeed, their PDI values indicated that they were not homogeneous and hence yielded a wide range of particle sizes. While the excipient used for manufacturing IDVPLGA NP was different than that for the nanocrystals, all NP were similar in size and charge distributions regardless of formulation technique; however nanocrystals were more homogeneous than PLGA particles and IDV-PLGA NP did not have a significant charge. Our previous studies have shown that particles with a weak charge are taken up to a lesser extent by MDM than particles with a stronger charge (positive or negative). ${ }^{15}$ Wet milled IDV NP were more negatively charged than homogenized and PLGA particles. RTV NP manufactured using all three techniques were similar in size and charge. ATV- and EFV-PLGA particles were more homogeneous than the ATV and EFV nanocrystals. Other physical characteristics were similar.

The use of MDM as a vehicle for drug delivery supports the idea that the cells have a preference for uptake in regard to drug type. MDM uptake of ATV NP manufactured by wet milling and homogenization was significantly higher than for those NP made from the other three drugs. In addition, the milled and the homogenized particles were taken up better by MDM than their respective PLGA formulations. RTV-PLGA NP was one exception that exhibited a high initial drug uptake. Drug retention was notable in ATV formulations particularly for the nanocrystals. ATV is one of the leading protease inhibitors currently prescribed in the clinic. ${ }^{31,32}$ Daily doses of RTV, another protease inhibitor, are used to boost ATV levels, thus a sustained-release formulation containing both ATV and RTV would help simplify ART regimens. ${ }^{32}$ Our NP manufactured with RTV showed that MDM retained the drug for up to 10 days. This sustained-release profile can be used to our advantage since it is a significant improvement over the current oral dosage regimen that requires patients to take one single-tablet daily. ${ }^{33}$ We used the sustained release of ATV and RTV NP to assess the efficacy of nanosuspensions since optimal adherence to conventional oral dosing regimens remains a clinical problem. Oral administration of antiretroviral 

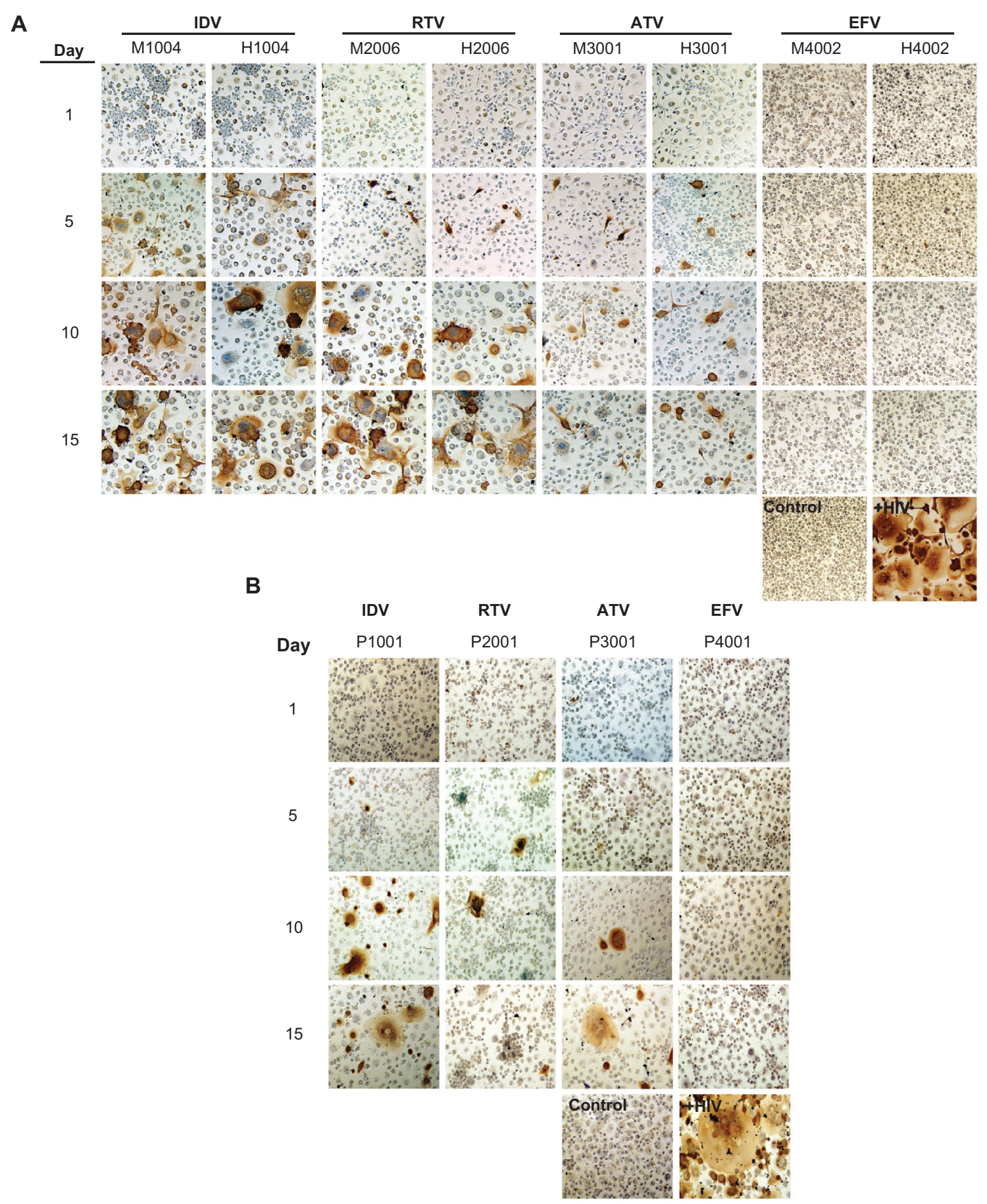

Figure 4 Antiretroviral efficacy of nanoART as determined by HIV-I 24 antigen staining.

Notes: Monocyte-derived macrophages (MDM) were loaded for 8 hours with IDV, RTV, ATV and EFV nanoparticles manufactured by (A) wet milling and homogenization or (B) sonication. MDM were infected with HIV-I ${ }_{\text {ADA }}$ on days I, 5, I0, and I5 post-loading and cultured for an additional 10 days. MDM were fixed and immunostained for expression of p24. Untreated, uninfected MDM served as negative controls (control), while MDM exposed to HIV-I ${ }_{A D A}$ but not treated with nanoART, served as positive controls (+HIV). Expression of viral p24 antigen was visualized by DAB chromogen (brown). Images are representative of $n=4$ determinations per treatment. (Magnification, $200 \times$ ).

Abbreviations: IDV, indinavir; RTV, ritonavir; ATV, atazanavir; EFV, efavirenz.

drugs has low biodistribution and elicits sustained viral sanctuaries that affect drug resistance. ${ }^{2,5}$ We reported previously that nanoformulations using macrophage-based drug delivery provide stable drug levels, and are able to reach viral sanctuaries when administered ex vivo. ${ }^{14}$ The drug taken up by MDM is active for a period of weeks as opposed to the conventional non-nanoformulated drugs, which are metabolized quickly, requiring the patients to follow daily dosing schedules. With conventional antiretroviral drugs administered orally, it is unlikely that suitable drug concentrations could be achieved at 

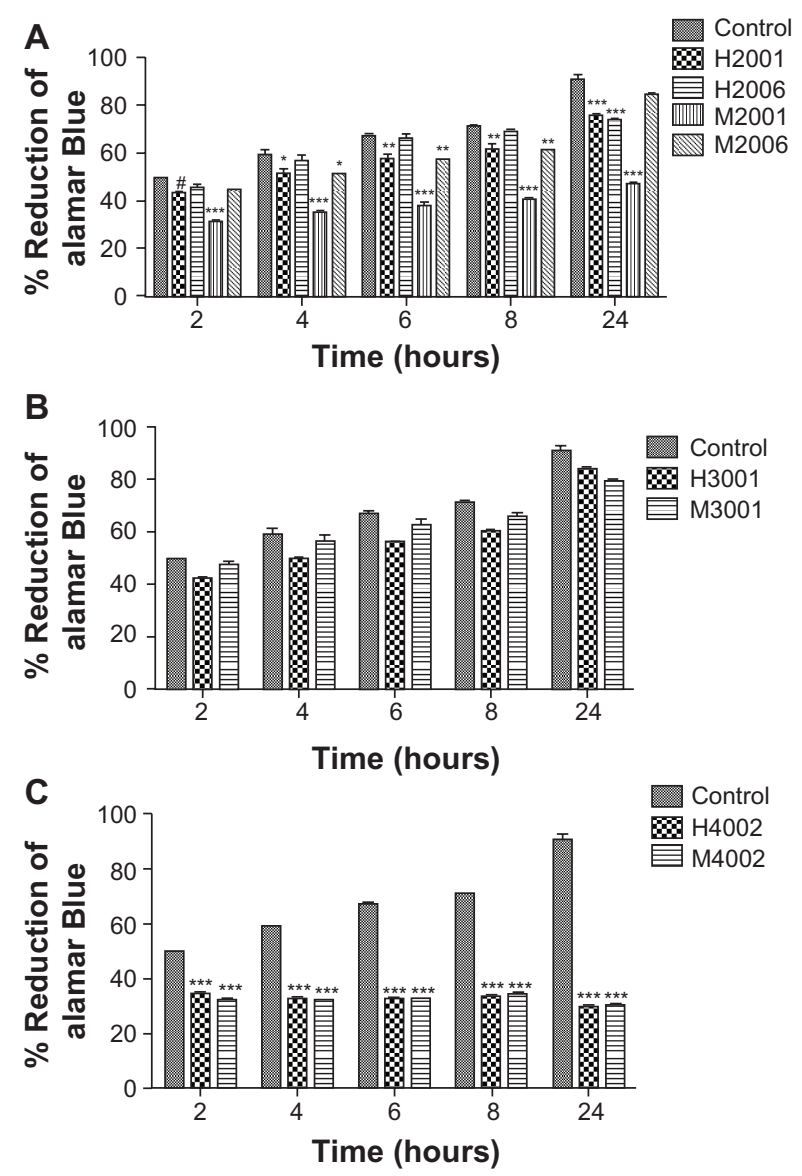

Figure 5 Effect of nanoART on macrophage viability.

Notes: Monocyte-derived macrophages (MDM) were loaded for 12 hours with (A) four nanoformulations of RTV (H200I, H2006, M200I, M2006), (B) two nanoformulations of ATV ( $\mathrm{H} 300 \mathrm{I}$ and $\mathrm{M} 300 \mathrm{I})$, or (C) two nanoformulations of EFV ( $\mathrm{H} 4002$ and M4002) at $0.1 \mathrm{mM}$. Controls consisted of untreated cells. Following drug loading, toxicity was assessed over 24 hours by alamarBlue ${ }^{\mathrm{TM}}$ assay. For each experimental condition, $n=3$. Figure shown is representative of two independent experiments. Significant differences are indicated: $\#(P<0.05), *(P<0.0 \mathrm{I})$, ** $(P<0.00 \mathrm{I})$, *** $(P<0.000 \mathrm{I})$

sites of HIV-infected tissues such as the brain or deep within the lymph nodes. NanoART has the potential to be effective in tissue sites where drug penetration by native drugs is limited. In this context, macrophages would carry drug-laden NP to tissue sites where conventional, oral drug administration could not reach. As noted MDM has potential advantages for ART delivery. One rests in the fact that antiretroviral efficacy may also be enhanced by subcellular delivery of ART into endosomal compartments where active viral replication ensues. ${ }^{34}$

The current studies suggest that all the manufacturing techniques would result in similar profiles for viral suppression by nanoART. Amongst the protease inhibitors, ATV was the most efficacious as viral suppression values were maintained at $\sim 70 \%$ and higher with all formulations manufactured using homogenization, milling, and sonication. Although, the suppression values were reduced to $24 \%$ by day 15 for RTV nanocrystals, this was significant since RTV is shown to boost the effect of ATV and has been reported to be effective and well-tolerated in HIV patients. ${ }^{35}$ The PLGA NP, although having higher viral suppression values, were held as less effective since their uptake was lower, reducing the chances to develop a sustained-release profile required to increase the drug-dosing interval. While EFV NP viral suppression values were at $95 \%$ and above for wet milled and homogenized formulations and above $90 \%$ for PLGA NP, the maximum uptake achieved using EFV was below $1.5 \mu \mathrm{g} / 10^{6}$ cells. The significant suppression seen for EFV as compared to ATV and RTV is based on the known nonnucleoside reverse transcriptase inhibition of antiretroviral replication. ${ }^{36}$ Nonetheless and although substantively efficacious, EFV has been reported to have commonly shown central nervous system side effects as well as population differences in pharmacokinetics. ${ }^{37}$ These comparisons of IDV-, ATV-, RTV-, and EFV-NP support the idea that MDM are a viable screening system that can be extended to applications of other drugs and infectious diseases.

Destache et $\mathrm{al}^{19}$ manufactured PLGA NP containing RTV, EFV, and lopinavir and reported concentration of antiretroviral drugs over a period of 28 days from NP incubated with peripheral blood mononuclear cells. The cellular drug level reported on day 4 for RTV was $2.5 \mu \mathrm{g}$ and for EFV was $10.6 \mu \mathrm{g}$; all formulations had drug concentrations $\geq 0.9 \mu \mathrm{g}$. The results suggested the use of PLGA NP for drug delivery using parenteral administration for prolonged release of drug. Since total drug loading of PLGA NP is $\sim 4 \%-5 \%(\mathrm{w} / \mathrm{w})$, prolonged parenteral administration of PLGA NP would result in exposure to high levels of excipients. With regard to PLGA-EFV NP the high level of drug in cells could result from more EFV being encapsulated in the NP as compared to the others although equal quantities of each drug were used in making the formulations. Cell uptake assays performed in those studies also worked on the assumption that aliquots sampled had cell equivalents. All together, the physical characteristics of PLGA NP for drug uptake and retention were limited.

To further support the use of cell-mediated drug delivery, nanocrystalline particles were used for toxicity studies. The ATV nanoparticles produced by the different manufacturing techniques did not significantly affect MDM viability, whereas, EFV nanoparticles affected MDM viability regardless of the method of manufacture. However, the manufacturing method did affect RTV nanoformulation cytotoxicities. Comparison of RTV nanoparticles prepared by homogenization and wet milling showed that the P188-coated RTV particles produced 
by wet milling induced more cytotoxicity than homogenized particles. Although wet milling and homogenization techniques produced NP that were similar in nature, the toxicity of the wet milled particles may be linked to the grinding media itself that contacts the drug and the excipient. The zirconium/ceramic media, although highly resistant to wear, has a tendency to break down over time because of the high milling speeds. This might contaminate the samples over time. Another possible source of contamination is wearing down of the metal screen that is used to prevent entry of the grinding media into the suspension during processing. Homogenization provided a non-contamination process, where no media is necessary to fractionate the drug particles. The homogenizer is controlled by a pneumatically-dynamic homogenizing valve with seals that are made of metal and do not use latex or rubber "O" rings. Any residual fluids after a complete cycle are removed from the instrument by blowing the system out with compressed air. This feature limits possible contaminants that could affect toxicity profiles.

We previously investigated the subcellular distribution of the drug particles in macrophages from the initial stage of cellular uptake to that of final release of the drug. NanoART proceeds through a sorting process into a recycling pathway after rapid clathrin-dependent internalization. ${ }^{34}$ Consequently, NP are in recycling endosomal compartments where a significant component of the virus' life cycle occurs. ${ }^{34,38,39}$ The data suggest that nanoART could enter the cell together with the virus and be located in identical subcellular destinations, further assisting in a targeted delivery to subcellular compartments. This could explain how nanoART are capable of suppressing HIV at low intracellular concentrations. ${ }^{11-13,15,20,34}$ Storage of the NP in recycling endosomes also helps avoid intracellular degradation and assists in the release of the NP at the cell surface. This method of particle trafficking may be analogous to HIV endocytic sorting, thus further assisting in restricting viral replication. After NP are recycled to the plasma membrane, they are intact and retain their antiretroviral properties. This strongly supports the role of MDM as "Trojan horses" for nanoART drug delivery and a mechanism for inhibiting viral replication at all sites of infection. These findings have established that macrophage-mediated drug delivery is a critical therapeutic option for an efficient and simplified drug regimen for the treatment of HIV.

\section{Conclusion}

NanoART have the potential to overcome the limitations in drug compliance, pharmacokinetics, and toxicities. We have shown that repackaging of clinically available antiretroviral medications into NP is a viable option for HIV treatment. We have also shown that a cell-based screening approach is an effective way to evaluate a wide variety of formulations. This will aid in the selection of superior performing formulations for future studies of long-acting injectables designed to increase the drug dosing interval and thus improve patient compliance.

\section{Acknowledgments}

The work was supported by the National Institutes of Health grants 1P01 DA028555, 2R01 NS034239, 2R37 NS36126, P01 NS31492, P20RR 15635, P01MH64570, 5P20 RR021937 (AVK), and P01 NS43985 (HEG) and from a research grant from Baxter Healthcare. The authors thank Alec Anderson, Landon Ehlers, Yudong Li, and Nathan Smith for their expert technical assistance. The authors would also like to thank Dr You Zhou of the University of NebraskaLincoln electron microscopy core facility for supplying the scanning electron microscopy images.

\section{Disclosure}

The authors report no conflicts of interest in this work.

\section{References}

1. Delaugerre C, Peytavin G, Dominguez S, et al. Virological and pharmacological factors associated with virological response to salvage therapy after an 8-week of treatment interruption in a context of very advanced HIV disease (GigHAART ANRS 097). J Med Virol. 2005;77(3):345-350.

2. Duval X, Peytavin G, Albert I, et al. Determination of indinavir and nelfinavir trough plasma concentration efficacy thresholds according to virological response in HIV-infected patients. HIV Med. 2004;5(4):307-313.

3. Hawkins T. Appearance-related side effects of HIV-1 treatment. AIDS Patient Care STDS. 2006;20(1):6-18.

4. Nowacek A, Gendelman HE. NanoART, neuroAIDS and CNS drug delivery. Nanomed. 2009;4(5):557-574.

5. Shehu-Xhilaga M, Tachedjian G, Crowe SM, Kedzierska K. Antiretroviral compounds: mechanisms underlying failure of HAART to eradicate HIV-1. Curr Med Chem. 2005;12(15):1705-1719.

6. Alos L, Navarrete P, Morente V, et al. Immunoarchitecture of lymphoid tissue in HIV-infection during antiretroviral therapy correlates with viral persistence. Mod Pathol. 2005;18(1):127-136.

7. Best BM, Letendre SL, Brigid E, et al. Low atazanavir concentrations in cerebrospinal fluid. Aids. 2009;23(1):83-87.

8. Murri R, Lepri AC, Cicconi P, et al. Is moderate HIV viremia associated with a higher risk of clinical progression in HIV-infected people treated with highly active antiretroviral therapy: evidence from the Italian cohort of antiretroviral-naive patients study. J Acquir Immune Defic Syndr. 2006;41(1):23-30.

9. Pathan SA, Iqbal Z, Zaidi SM, et al. CNS drug delivery systems: novel approaches. Recent Pat Drug Deliv Formul. 2009;3(1):71-89.

10. Varatharajan L, Thomas SA. The transport of anti-HIV drugs across blood-CNS interfaces: summary of current knowledge and recommendations for further research. Antiviral Res. 2009;82(2):A99-A109. 
11. Nowacek AS, Balkundi S, McMillan J, et al. Analyses of nanoformulated antiretroviral drug charge, size, shape and content for uptake, drug release and antiviral activities in human monocyte-derived macrophages. J Control Release. 2011;150(2):204-211.

12. Nowacek AS, McMillan J, Miller R, Anderson A, Rabinow B, Gendelman HE. Nanoformulated antiretroviral drug combinations extend drug release and antiretroviral responses in HIV-1-infected macrophages: implications for neuroAIDS therapeutics. J Neuroimmune Pharmacol. 2010;5(4):592-601.

13. Dou H, Destache CJ, Morehead JR, et al. Development of a macrophage-based nanoparticle platform for antiretroviral drug delivery. Blood. 2006;108(8):2827-2835.

14. Dou H, Grotepas CB, McMillan JM, et al. Macrophage delivery of nanoformulated antiretroviral drug to the brain in a murine model of neuroAIDS. J Immunol. 2009;183(1):661-669.

15. Nowacek AS, Miller RL, McMillan J, et al. NanoART synthesis, characterization, uptake, release and toxicology for human monocyte-macrophage drug delivery. Nanomedicine (Lond). 2009;4(8):903-917.

16. Balkundi S, Nowacek AS, Roy U, Martinez-Skinner A, McMillan J, Gendelman HE. Methods development for blood borne macrophage carriage of nanoformulated antiretroviral drugs. J Vis Exp. 2010;46. pii: 2460. doi: 10.3791/2460.

17. Bressani RF, Nowacek AS, Singh S, et al. Pharmacotoxicology of monocyte-macrophage nanoformulated antiretroviral drug uptake and carriage. Nanotoxicology. 2010;5:592-605.

18. Corrias F, Lai F. New methods for lipid nanoparticles preparation. Recent Pat Drug Deliv Formul. 2011;5(3):201-213.

19. Destache CJ, Belgum T, Goede M, Shibata A, Belshan MA. Antiretroviral release from poly(DL-lactide-co-glycolide) nanoparticles in mice. J Antimicrob Chemother. 2010;65(10):2183-2187.

20. Dou H, Morehead J, Destache CJ, et al. Laboratory investigations for the morphologic, pharmacokinetic, and anti-retroviral properties of indinavir nanoparticles in human monocyte-derived macrophages. Virology. 2007;358(1):148-158.

21. Mainardes RM, Evangelista RC. PLGA nanoparticles containing praziquantel: effect of formulation variables on size distribution. Int J Pharm. 2005;290(1-2):137-144.

22. Rabinow BE. Nanosuspensions in drug delivery. Nat Rev Drug Discov. 2004;3(9):785-796.

23. Shegokar R, Jansch M, Singh KK, Muller RH. In vitro protein adsorption studies on nevirapine nanosuspensions for HIV/AIDS chemotherapy. Nanomedicine. 2011;7(3):333-340.

24. Takatsuka T, Endo T, Jianguo Y, Yuminoki K, Hashimoto N. Nanosizing of poorly water soluble compounds using rotation/revolution mixer. Chem Pharm Bull (Tokyo). 2009;57(10):1061-1067.

25. Nakarani M, Patel P, Patel J, Murthy RS, Vaghani SS. Cyclosporine a-nanosuspension: formulation, characterization and in vivo comparison with a marketed formulation. Sci Pharm. 2010;78(2):345-361.
26. Niwa T, Miura S, Danjo K. Universal wet-milling technique to prepare oral nanosuspension focused on discovery and preclinical animal studies - Development of particle design method. Int J Pharm. 2011;405(1-2):218-227.

27. Destache CJ, Belgum T, Christensen K, et al. Combination antiretroviral drugs in PLGA nanoparticle for HIV-1. BMC Infect Dis. 2009;9:198

28. Reddy MK, Wu L, Kou W, Ghorpade A, Labhasetwar V. Superoxide dismutase-loaded PLGA nanoparticles protect cultured human neurons under oxidative stress. Appl Biochem Biotechnol. 2008;151(2-3):565-577.

29. Gendelman HE, Orenstein JM, Martin MA, et al. Efficient isolation and propagation of human immunodeficiency virus on recombinant colony-stimulating factor 1-treated monocytes. J Exp Med. 1988;167(4):1428-1441.

30. Kalter DC, Nakamura M, Turpin JA, et al. Enhanced HIV replication in macrophage colony-stimulating factor-treated monocytes. J Immunol. 1991;146(1):298-306.

31. Swindells S, DiRienzo AG, Wilkin T, et al. Regimen simplification to atazanavir-ritonavir alone as maintenance antiretroviral therapy after sustained virologic suppression. JAMA. 2006;296(7): 806-814.

32. Swindells S, Flexner C, Fletcher CV, Jacobson JM. The critical need for alternative antiretroviral formulations, and obstacles to their development. J Infect Dis. 2011;204(5):669-674.

33. Deeks ED, Perry CM. Efavirenz/emtricitabine/tenofovir disoproxil fumarate single-tablet regimen (Atripla(R)): a review of its use in the management of HIV infection. Drugs. 2010;70(17):2315-2338.

34. Kadiu I, Nowacek A, cMillan J, Gendelman HE. Macrophage endocytic trafficking of antiretroviral nanoparticles. Nanomedicine. 2011;6(1):25-42.

35. Johnson M, Grinsztejn B, Rodriguez C, et al. Atazanavir plus ritonavir or saquinavir, and lopinavir/ritonavir in patients experiencing multiple virological failures. Aids. 2005;19(7):685-694.

36. Martins S, Ramos MJ, Fernandes PA. The current status of the NNRTI family of antiretrovirals used in the HAART regime against HIV infection. Curr Med Chem. 2008;15(11):1083-1095.

37. Haas DW, Ribaudo HJ, Kim RB, et al. Pharmacogenetics of efavirenz and central nervous system side effects: an Adult AIDS Clinical Trials Group study. Aids. 2004;18(18):2391-2400.

38. Murray JL, Mavrakis M, McDonald NJ, et al. Rab9 GTPase is required for replication of human immunodeficiency virus type 1, filoviruses, and measles virus. J Virol. 2005;79(18):11742-11751.

39. Varthakavi V, Smith RM, Martin KL, et al. The pericentriolar recycling endosome plays a key role in Vpu-mediated enhancement of HIV-1 particle release. Traffic. 2006;7(3):298-307.
International Journal of Nanomedicine

\section{Publish your work in this journal}

The International Journal of Nanomedicine is an international, peerreviewed journal focusing on the application of nanotechnology in diagnostics, therapeutics, and drug delivery systems throughout the biomedical field. This journal is indexed on PubMed Central, MedLine, CAS, SciSearch $\AA$, Current Contents ${ }^{\circledR} /$ Clinical Medicine,

\section{Dovepress}

Journal Citation Reports/Science Edition, EMBase, Scopus and the Elsevier Bibliographic databases. The manuscript management system is completely online and includes a very quick and fair peer-review system, which is all easy to use. Visit http://www.dovepress.com/ testimonials.php to read real quotes from published authors. 